\title{
PULMONARY SEQUESTRATION*
}

\author{
BY
}

\author{
P. TALALAK \\ From the Surgical Department, the Children's Hospital, Bremen
}

Pulmonary sequestration is a developmental anomaly of the pulmonary and cardiovascular system. It consists of dissociated, non-functioning lung tissue which is supplied by aberrant arteries and has no communication with the normal bronchial system. It may be divided into two types, intralobar and extralobar. This anomaly has become an important and interesting clinical problem in the past 10 years. Most of the cases were diagnosed and treated as lung abscess, empyema or bronchiectasis because the real diagnosis had been overlooked.

The number of reported cases has increased rapidly during the last 10 years. Nevertheless in 1958 Eaker, Hannon and French discovered only 104 cases; Warner, Britt and Riley (1958) reported three cases. In addition I would like to report three cases from the Bremen Children's Hospital.

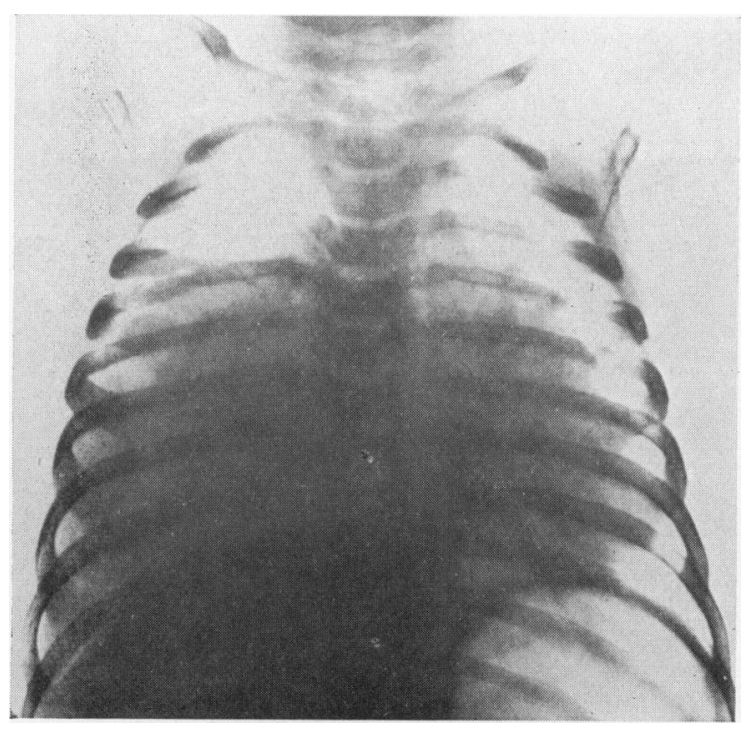

Fig. 1.-Case 1. Large opacity in right hemithorax with displacement of heart and mediastinum to left side.

\footnotetext{
* A paper read at a meeting of the British Association of Paediatric Surgeons held in Liverpool in June, 1959.
}

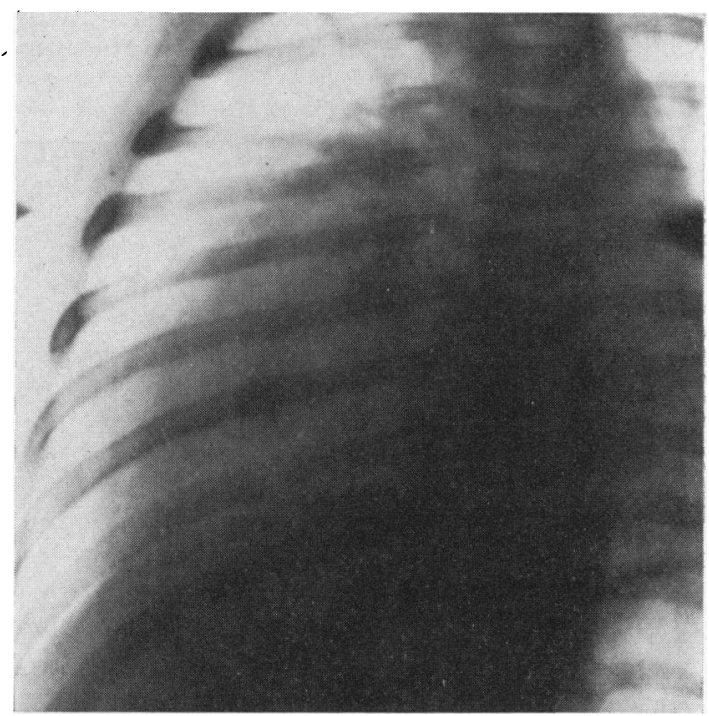

FIG. 2.-Case 1. On rotation shadow is seen to lie in posterior region of thorax. An area of calcified density is visualized.

\section{Case Reports}

Case 1. A 6-month-old girl was admitted because of high fever. A radiograph (Fig. 1) showed an area of opacity in the right lung field. On rotation of the patient it was seen to be in the lower posterior region of the chest wall, and an area of calcified density was visualized (Fig. 2). The heart and mediastinum were shifted to the left side. At operation there was a mass about the size of two fists arising from the right lower lobe, which could not be detached from the lung or diaphragm. Therefore the lower lobe was resected. As we tried to free the adhesion from the diaphragm an accessory artery the size of a pencil was seen arising from the thoracic aorta and running to the lower lobe. Moreover there was an opening about $2.5 \mathrm{~cm}$. in diameter on the right side of the diaphragm containing a mushroom-shaped protrusion of liver, which communicated with the lower lobe through the adhesion tissue.

Unfortunately the child died immediately after the operation. The macroscopical specimen showed a small cystic formation of the whole lower lobe, part of which became a solid yellow area. There was normal lung tissue only at the anterior margin of the lobe. At the 
base of the lung there was a communication of the arterial branch into the liver through the opening in the diaphragm. Histological examination showed that the cysts were lined with bronchial epithelium (some ciliated) containing cellular debris and desquamated epithelium.

According to the reported cases in the literature, this case is a rare one. Besides a right intralobar pulmonary sequestration there was a defect of the diaphragm and communication between the sequestration and the liver.

Case 2. A 14-year-old boy without symptoms was admitted because of a tumour shadow in the lower part of the right thorax, which had been found accidentally two years previously on serial radiological examination. There was a dense oval shadow in the posterior part of the right lower lobe (Fig. 3a, b), which revealed a fluid level on the cranial side and had two round protrusions. Bronchographic examination (Fig. 3c) showed that the bronchus segment no. 6 was displaced upward, and no. 9 and no. 10 forward. At operation the lower lobe was seen to be composed of many small cystic tumours. It was impossible to dissect these masses from the lower lobe and therefore lobectomy was performed. The pulmonary ligament contained an abnormal artery about the size of a pencil from the aorta to the lower lobe. There was no post-operative complication and the radiograph showed a satisfactory result (Fig. 3d).

Histological examination showed an area of extensive widening of the bronchi with two large cystic formations in the lower lobe. They were irregularly lined with simple

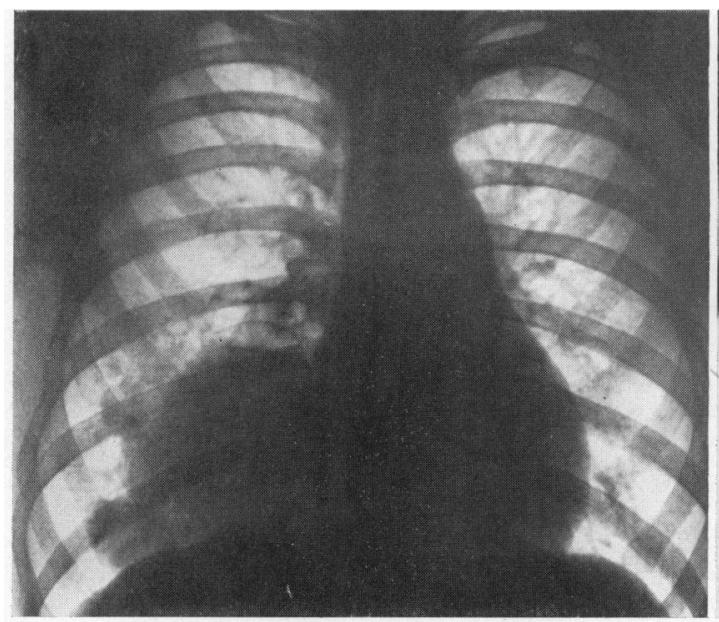

(a)

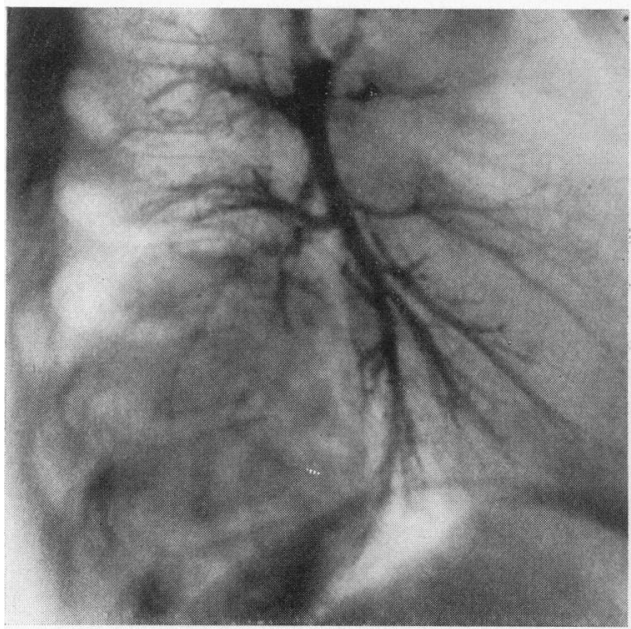

(c)

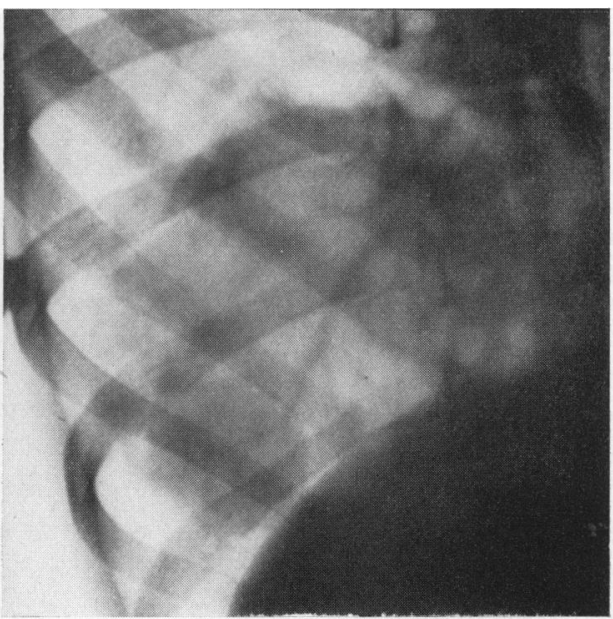

(b)

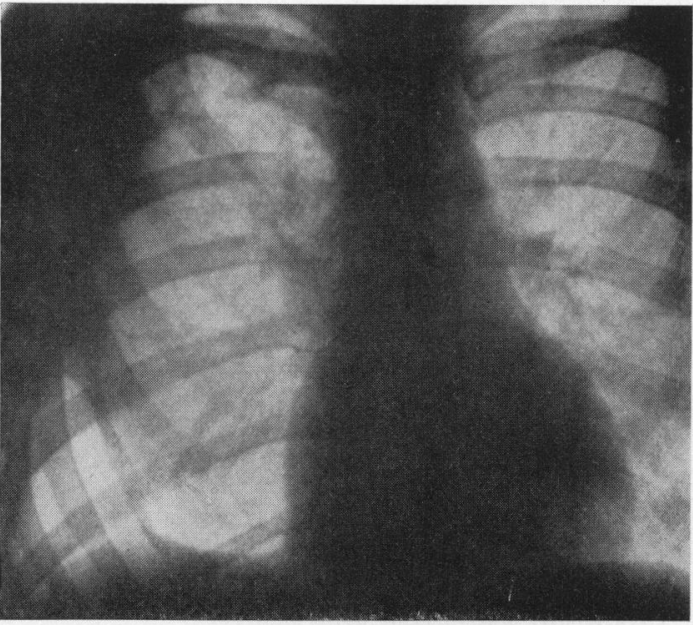

(d)

Fig. 3.-Case 2. (a and b) Dense oval shadow in lower posterior part of right hemithorax with presence of two round protrusions and a small fluid level; (c) displacement of bronchi of lower lobe ; (d) post-operative radiograph. 
columnar and some stratified columnar epithelium, and contained abundant desquamated epithelium, cellular debris and leucocytes. The surrounding area was composed of respiratory epithelium. At the margin of the lobe there was normal lung tissue with functional alveoli.

Case 3. The last patient was a child 14 days old, admitted because she had refused to feed for the past two to three days. Clinical examination revealed the presence of a left diaphragmatic hernia, which was confirmed by radiographs (Fig. 4). Laparotomy was performed, and the stomach, small intestine, part of the

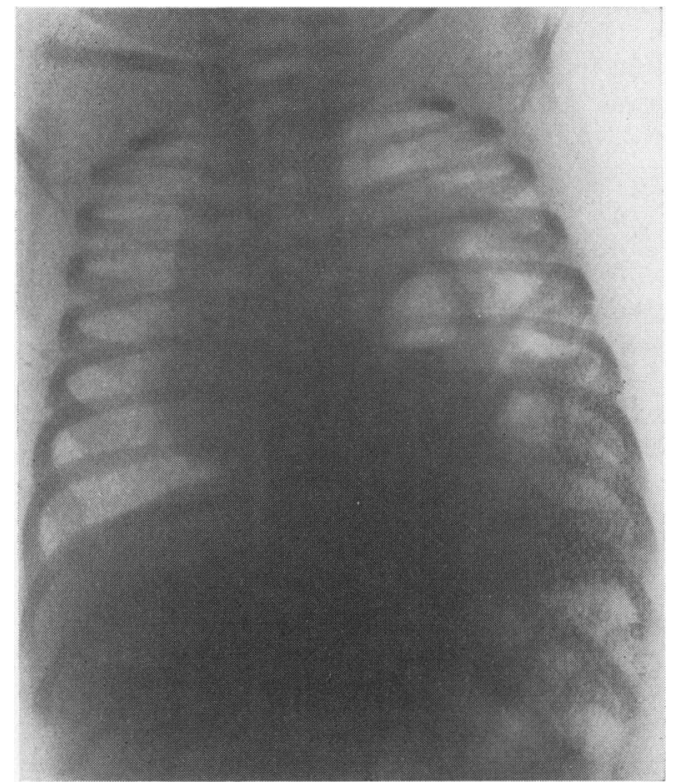

FIG. 4.-Case 3. Shows presence of diaphragmatic hernia on left.

colon and spleen had protruded into the left thorax through a large opening in the diaphragm. They were carefully pulled down. On opening the hernial sac the left lower lobe was found to be rather hypoplasic, but functioning well. On further examination of the left thorax, a small accessory pulmonary lobe-like mass in the posterior costo-diaphragmatic angle was seen. The consistency was that of atelectatic tissue but there was no connexion with the adjacent lung.

Fig. 5 shows the presence of the eventration and its relationship to surrounding structures. Behind the hernial sac is the medial pedunculated accessory lung lobe, which contains an artery arising from the aorta. The mass was removed and the diaphragm was reconstructed by means of the hernial sac membrane. Uncomplicated post-operative progress was verified radiologically (Fig. 6). The child was completely cured.

Microscopical examination showed the presence of bronchi, bronchioles and normal respiratory epithelium.

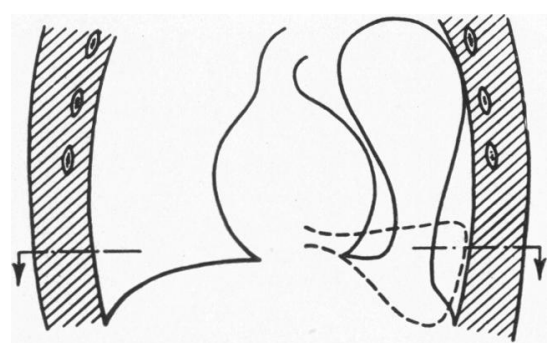

Fig. 5.-Case 3. Diagram showing relationship between diaphragm, hernial sac and accessory lobe.

There was extensive widening of many bronchi. Some groups of alveoli contained foam cells (Fig. 7).

\section{Discussion}

Many theories have been advanced about the pathogenesis of this anomaly. The 'traction theory' of Pryce (1946) seems to be the most acceptable. He states that the arterial component is the primary and the malformation of the pulmonary component is secondary. As a result of the persistence of an

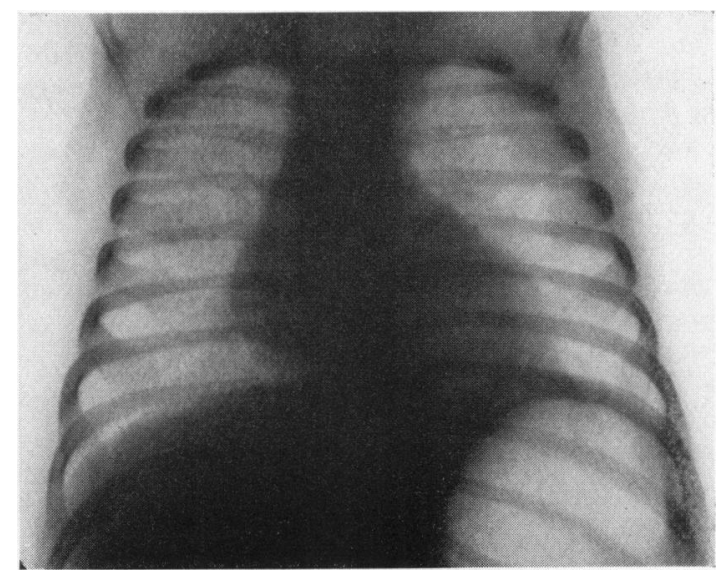

Fig. 6.-Case 3. Twenty-two days after operation. 


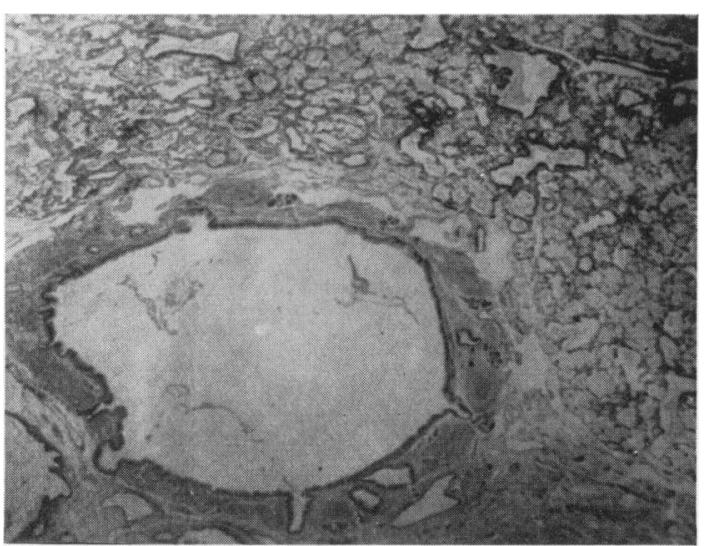

Fig. 7.-Case 3. Histological section showing bronchus, bronchioles and alveoli.

aberrant artery, which supplied a part of the primitive lung lobe, a vascular traction from this lung lobe occurs. The consequence is the dissociation of a piece of pulmonary tissue developed as a sequestration. On the other hand there is the 'fraction theory' which states that pulmonary sequestration develops by abnormal dissociation of a small primitive lung component which seeks an arterial supply from the visceral branch of the aorta itself.

Pulmonary sequestration is composed of respiratory epithelium and ramified bronchi or bronchiectasis without communication with the normal bronchial system. Intralobar sequestration occurs most often in the lower lobe, on the postero-medial aspect. The cases of Cohn and Hopeman (1955), and Warner et al. are the only exceptions, because these were found in the upper lobe. Extralobar sequestration is a completely dissociated or ectopic lobe, which can be found above or below the diaphragm. Only in the case of Gruber (1914) was the peduncle attached to the lateral thoracic wall.

The commonest location of extra-lobar sequestration is the left thoracic side and it is related to a diaphragmatic hernia, as in our third case. It is also found in the pericardium and in the abdomen. Location in the right side is rare. Associated anomalies are found, such as congenital absence of pericardium and communication with the oesophagus. Intralobar sequestration is predominant on the right side and has no relation to other congenital malformations.

With the exception of the complications there is no definite clinical feature of this condition. Sometimes there might be a history of chronic respiratory tract infection, such as productive cough, fever, pleuritic pain, etc. In only a few cases were the patients diagnosed pre-operatively. In most of them the sequestration was found accidentally on radiological examination or at operation. No typical feature appears on the radiograph. However, in a chest film which shows an opacity in the lower lobe and displacement of the bronchi with normal pulmonary findings, we should at least consider this condition. Bronchography is important in the diagnosis, but differentiation from a lung cyst or tumour is not easy. Only in a case of infected pulmonary sequestration can we perhaps see the communication between both bronchial systems on bronchography. Aortography has been suggested by some authors.

Infection of an intralobar sequestration is the commonest complication and is often found. The mode of infection is supposed to be haematogenous. Tuberculosis, malignant change and haemorrhage have also been reported.

The only treatment is operative extirpation of the sequestration. Most authors prefer lobectomy for the intralobar sequestration because removal of the sequestration alone is possible only in exceptional cases. After conservative operation the focus of infection remains in the lung and can spread at any time. Moreover, communication of both pulmonary and aberrant circulations after conservative operation has been observed and an A-V shunt should occur.

I am grateful to Professor Dr. F. Rehbein of Bremen for permission to publish this paper.

REFERENCES

Cohn, R. and Hopeman, A. (1955). Stanford med. Bull., 13, 361. Eaker, A. B., Hannon, J. L. and French, S. W. (1958). Am. J. Surg., 95, 31 .

Gruber, G. B. (1914). Beitr, path. Anat., 59, 491.

Pryce, D. M. (1946). J. Path. Bact., 58, 457. Warner, C. L., Britt, R. L. and Riley, H. D., Jr. (1958). J. Pediat., 